\title{
Mechanisms of Covid-Induced Heart Failure
}

\author{
Frank Bunks ${ }^{1}$ and Roel Mercado ${ }^{1 \#}$ \\ ${ }^{1}$ Saint Joseph High School, Metuchen, NJ, USA \\ \#Advisor
}

\section{ABSTRACT}

Sars-Cov-2 binds to ACE-2 receptor, resulting in excess amount of ACE-1 dependent angiotensin production. SarsCov-2 has four stages of symptoms, $80 \%$ if symptomatic patients have been shown to only suffer a "mild" disease course, meanwhile $20 \%$ endure stage 3 , which is characterized by conditions such as ARDS, shock, and multiorgan failure. The binding of Covid to ACE receptors promotes the conversion of angiotensin 1 to angiotensin 2, constricting blood vessels, leading to thrombosis, lack of oxygen, and Ischemia. In Cardiac injury, the Covid receptor becomes represented in the heart, inducing in a pro-inflammatory increase, high cytokine concentrations, myocyte cardiac apoptosis, cardiac arrythmia, cardiac fibrotic tissue, myocarditis, and Heart failure. Cytokine storms associated with production of S1P, TNF activation of MMPs prompts myocardial depression, dilation of LV, and Heart failure. Covidinduced Sepsis corresponds with dysregulated host immune responses, increased pro-inflammatory mediators, fluid leakage, increase in cGMP, LV dysfunction, and arrythmia. Blood clots in the capillaries surrounding the kidneys generate renal impairment. Progression of renal impairment generates a state of systemic inflammation. Increased sodium content in the body results in elevated plasma blood and uncontrolled hypervolemia. Emotional and physical stresses during Sars-Cov-2 induce blood gas changes, angiotensin converting enzyme imbalance, and immune/inflammatory factors. Overactivation of the SNS induces Takotsubo syndrome. In ARDS, fluid leak in the membrane gas exchange region of the lung results in vascular remodeling. Inducing further vascular remodeling as part of the body's response to hypoxia. The constant vascular remodeling triggers RHF.

\section{Introduction}

\section{Overview of Covid-19}

In late 2019, a new strain of Coronavirus, Sars-Cov-2, was discovered. This virus resulted in an almost immediate global pandemic, fully shutting down countries like Italy, Germany, and Sweden. Currently, Sars-Cov-2 has a recorded over 194 million cases with more than 4.16 million deaths. According to the Sepsis alliance, a person with no existing conditions only has a $0.9 \%$ mortality rate. Meanwhile, someone with cancer has a $5.6 \%$, abnormally high blood pressure is $6 \%$, chronic respiratory disease is $6.3 \%$, diabetes is $7 \%$, and cardiovascular disease is $10.5 \%$.

When Sars-Cov-2 enters the body, it binds to the ACE-2 receptor, instigating in a decrease of its regulation over the ACE-1 receptor. The ACE-2 receptor is known to participate in "feed-back regulation" of the renin-angiotensin system through impeding ACE-1 production. However, since Covid binds to the receptor, ACE- 2 can no longer participate in its regulation, resulting in an excess amount of ACE-1dependent angiotensin production, causing noxious vasoconstrictive, and pro-inflammatory and prooxidative effects on the patient's vascular system. This Covid-19 induced ambulance of the ACE-1/ACE-2 receptors brings about the ACE-1 "shedding" phenomenon, believed to augment local inflammation. 


\section{Overview of Heart layers and Heart failure}

The heart is surrounded by three layers: Epicardium, myocardium, and endocardium. Epicardium consists of the Fibrous pericardium and Serous pericardium. The serous pericardium contains the Visceral pericardium, parietal pericardium, and the pericardial cavity. The pericardial cavity contains the pericardial fluid which is responsible for cushioning the outer and inner layers of the pericardium. The fluid allows the heart to expand and contract.

The heart is a four-chamber organ consisting of the right atrium, right ventricle, left atrium, and left ventricle. It also consists of the inferior vena cava which carries blood from the legs, abdomen, back, pelvis to the atrium. The left ventricle, the main powerhouse of the heart, pumps blood through the systemic circulation. The right ventricle, which conveys the right branch of the atrioventricular bundle (part of the heart's conduction system), contains deoxygenated blood. The atrioventricular bundle is responsible for making sure that the papillary muscles contract before the rest of the ventricle. As a result, the papillary muscles generate tension on the chordae tendineae, preventing the valve flaps and blood from being forced back into the atria.

Heart failure is a condition characterized by the hearts inadequate ability to pump blood. The mortality of heart failure increases by the time a person has the condition. According to the Framingham Heart Study, the mortality rate is $10 \%$ for the first 30 days, $20-30 \%$ at one year, and $45-60 \%$ at four or more years. There are four stages to Heart failure: stage A, pre-heart failure, is when a person has an increased chance of developing this condition due to family history. Stage B is characterized by a patient diagnosed with Left Ventricular dysfunction without the symptoms of heart failure. During Stage C, a patient has been diagnosed with heart failure and is exhibiting symptoms, or previously exhibited symptoms; these symptoms may go away with treatment. Stage D Heart failure is characterized by advanced symptoms that do not resolve with treatment.

\section{Key Words}

Heart Failure: Heart cannot adequately pump blood

Ischemia: Inadequate blood supply to an organ, especially the heart

Thrombosis: Formation of blood clots

Myocarditis: Inflammation of the myocardium of the Heart

Inflammatory Mediator: Molecules that function in promoting inflammatory response through the release of special substances

Myocardial Depression: Abnormal cardiomyocyte function, defined by contractile dysfunction and/or impaired relaxation such as inflammatory cytokines

Left Ventricular Dysfunction: A deformation and/or damage of the Left Ventricle that results in an abnormal enddiastolic volume

Septic Shock: Widespread infection resulting in organ failure and Low blood pressure

Renal Impairment: Kidneys lose ability to remove waste and balance fluid

Volume Overload: Expansion of extracellular fluid volume

RAAS (Renin-Angiotensin-Aldosterone system): hormone system in the body that is essential for the regulation of blood pressure and fluid balance

Sympathetic Activation: Response of the Sympathetic nervous system in stressful situations

Stress cardiomyopathy: Intense emotional and/or physical stress causing acute reversible Cardiac Dysfunction ARDS (Acute respiratory Disorder): Respiratory failure characterized by rapid onset of widespread inflammation in the lungs causing fluid to collect in the lungs' air sac, depriving the organs of oxygen

Pulmonary Hypertension: Type of high blood pressure that causes an increase in the muscles in the walls of blood vessels in the lungs

Right Heart Failure (RHF): Deformation and dysfunction of the right heart structures in response to increase vascular resistance 


\section{Pathways}

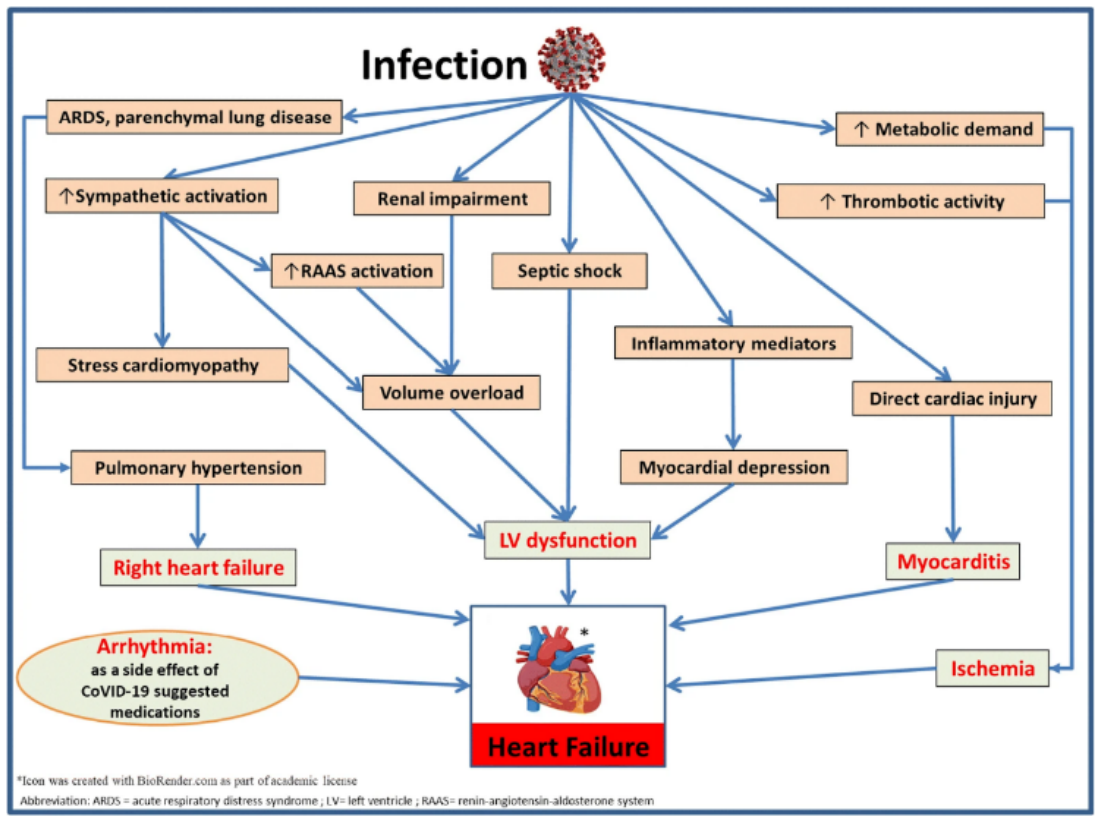

Fig.1 Covid-19 pathway to Heart failure. Reprinted by permission from Springer Nature: Springer, Heart Failure Reviews, (Bader et al, 2020), "Heart failure and COVID-19," F. Bader, Y. Manla, B. Atallah, R. Starling, Copyright 2020.

\section{Metabolic Demand}

A person's metabolic health is determined by the proper functioning of the metabolic processes coordinated by multiple physiological systems. A disturbance in those systems can cause a defective organismal metabolic process, resulting in the decline of the patient's metabolic health. This makes poor metabolic health a leading risk factor in the development and fatality of Sars-Cov-2. Examples of the risk factors in poor metabolic health include Type 2 diabetes, Obesity, and Hypertension. Individuals who are high-risk usually have some sort of condition that impairs their immune and antiviral responses to Covid and/or other viral and bacterial illnesses.

Certain risk factors such as Diabetes have been known to increase the risk of pulmonary fibrosis and reduce respiratory function. Obesity has been known to cause physical stress on ventilation through the obstruction of the Diaphragm excursion. Also, Hypertension can induce in blood clots such as Deep Vein Thrombosis as well which can cause arrhythmia.

A significant factor when looking at how Covid effects metabolic health is whether the patient is asymptomatic or symptomatic. Asymptomatic individuals will maintain their health during the disease through the representation of the health phenotype. Symptomatic individuals will exhibit symptoms after a pre-symptomatic phase. Statistics show that $80 \%$ of those with symptoms will display a mild disease "course", meanwhile $20 \%$ will advance to more severe stages including ARDS, Pneumonia, Septic Shock, etc. There are 4 stages of symptoms and recovery in Sars-Cov-2. 


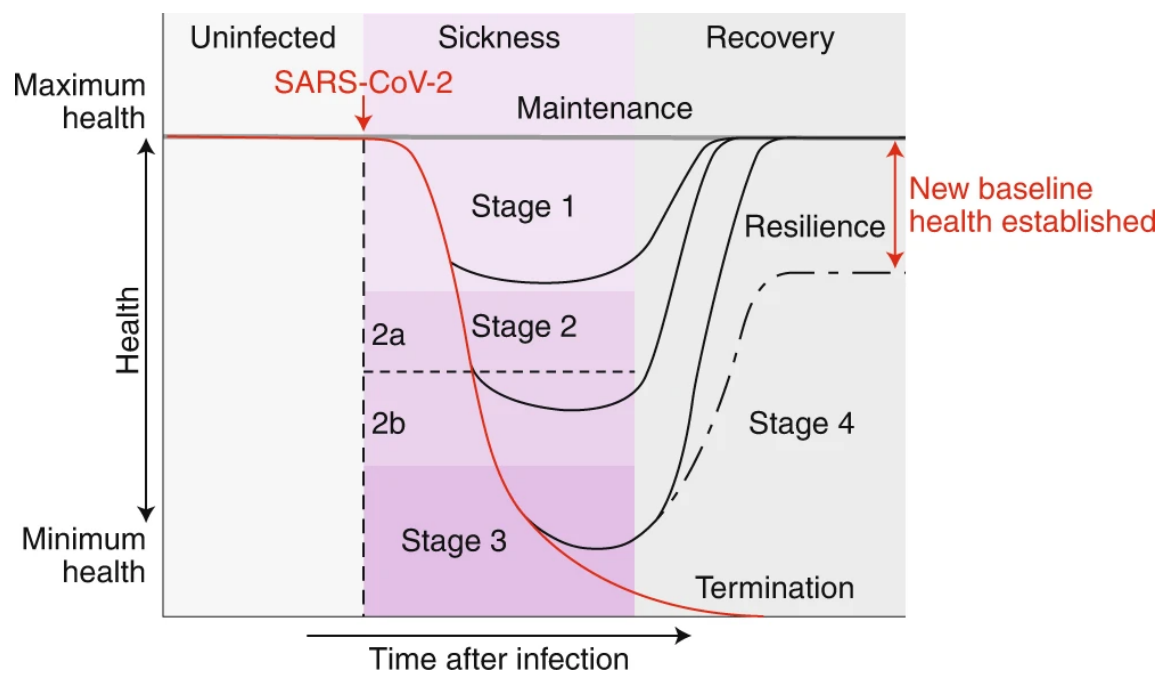

Fig. 2 Graph portrays the three stages of Metabolic Health during Sars-Cov-2. The further covid progressed in stages, the lower the patient's health became. The New baseline health is the difference between a person's previous maximum health (before Sars-Cov-2) and their current health after recovery. Stage 4 is the recovery stage, which is called resilience. Reprinted by permission from Springer Nature: Nature, nature metabolism, (Ayres, 2020), "A metabolic handbook for the COVID-19 pandemic," J. S. Ayres, Copyright 2020.

Patients with stage 1 symptoms will present with fever, dry cough, and any other general sense of discomfort, lack of interest in activities, fatigue, etc. Patients who progress to stage 2 will go through two different mini stages. Stage $2 a$ is characterized by pneumonia without hypoxia, meanwhile, stage $2 b$ is characterized by pneumonia with hypoxia. Stage 3 is considered to be the fatal stage of Covid. Patients in this stage develop conditions such as Acute Respiratory Disease Syndrome, Shock, and multiorgan failure. The final stage, stage 4, is the "recovery" stage. All patients, whether they went through stages 2(a)-3, go through stage 4. During stage 4, most patients will recover and return to their maximum health. However, certain patients will not fully recover, establishing a new baseline health. Patients who recover during stage 4 exhibit a resilience phenotype.

Critical phases of Covid-19 are induced by excess inflammatory responses, patients with compromised metabolic health will have an increased chance that response reaches the pathogenic level, as well as cause physiological harm. Examples include the process by which diabetes causes diabetic kidney disease leading to a decline in kidney function. Patients with compromised metabolic health due to issues such as excess inflammatory responses are at greater risk of hyperglycemia and hypertension damage to blood vessels resulting in an altered heart structure. A patient who suffers from hypertension develops a substantial risk for Ischemia, which can cause Heart failure.

\section{Thrombosis}

Research done on rats shows that the Sars-Cov-2 virus instigates in an increased Thrombosis, specifically in the Inferior Vena Cava. When the virus binds to the ACE-2 receptor enzyme, the expression of the enzyme is decreased. The ACE receptor inhibitor binds to the angiotensin receptors converting angiotensin 1 to angiotensin 2 . This conversion promotes an exaggerated constriction of the blood vessels. This reduces fibrinolysis, ensuring a major increase in blood clots. Thrombosis can lead to mainly deadly diseases, most commonly Ischemia.

The blockage of the coronary flow caused by Thrombosis causes a disturbance of the oxygen supplements, glucose supplements, and a washout in the affected tissue. Thrombosis also leads to a sudden increase in $\mathrm{K}^{+}$extracellular concentration, causing cardiac arrhythmia. Cardiac Arrythmia causes a diastolic dysfunction, prompting Left Ventricular Dysfunction and Heart failure. 


\section{Direct Cardiac Injury}

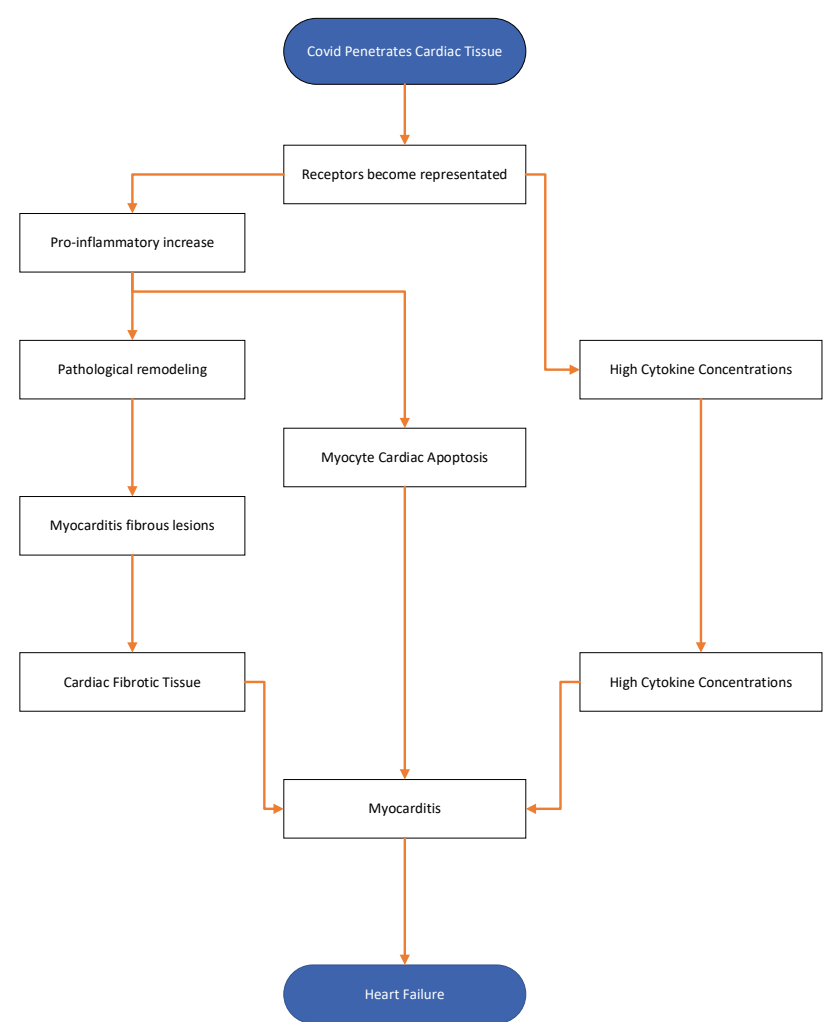

Fig. 3 Highlights the major steps in which Sars-Cov-2 causes Heart failure through Direct Cardiac Injury, specifically myocarditis.

Direct Cardiac Injury is when the tissue surrounding the heart (as well as the heart walls) becomes inflamed. A direct cardiac injury is considered to be an immediate and direct cause of Covid because the virus infects the heart tissue.

When Sars-Cov-2 infects the heart, its receptors become represented in the myocardial cells, resulting in both a sudden pro-inflammatory increase and a high cytokine concentration. The sudden increase in pro-inflammatory mediators is known to have an association with systemic inflammation. The systemic inflammation can have two different types of effects on the heart. First, it can cause contractile dysfunction as well as myocyte cardiac apoptosis, this condition is considered essential to causing Heart failure in a patient, leading to myocarditis; or it can lead to pathological remodeling of the extracellular matrix, leading to myocardial fibrous lesions, causing cardiac fibrotic tissue, leading to myocarditis. The myocardial fibrous lesions caused by the pathological remodeling of the extracellular matrix induces an increased collagen type 1 deposition (increases the strength of the wound), as well as cardiac fibroblast activation and differentiation into myofibroblasts (contributes to many different properties of the myocardium). This increase in collagen and fibroblasts can bring about cardiac fibrotic tissue.

Another possible result of the Sars-Cov-2 being represented in Cardiac cells is high cytokine concentrations. The high cytokine concentrations result in cytokine-induced organ dysfunction, specifically cardiac arrythmia, through an inflammatory immune dysregulation. Cardiac arrythmia and myocarditis work simultaneously. Cardiac arrythmia is the heart's inability to keep a good electrical signal.

Myocarditis is the heart's reduced ability to pump, resulting in an abnormal heartbeat and heart failure. Myocarditis is either caused by inflammation of pro-inflammatory mediators: myocyte cardiac apoptosis, or cardiac fibrosis tissue. Myocarditis can also be caused by high cytokine concentrations resulting in an abnormal. 


\section{Inflammatory Mediators}

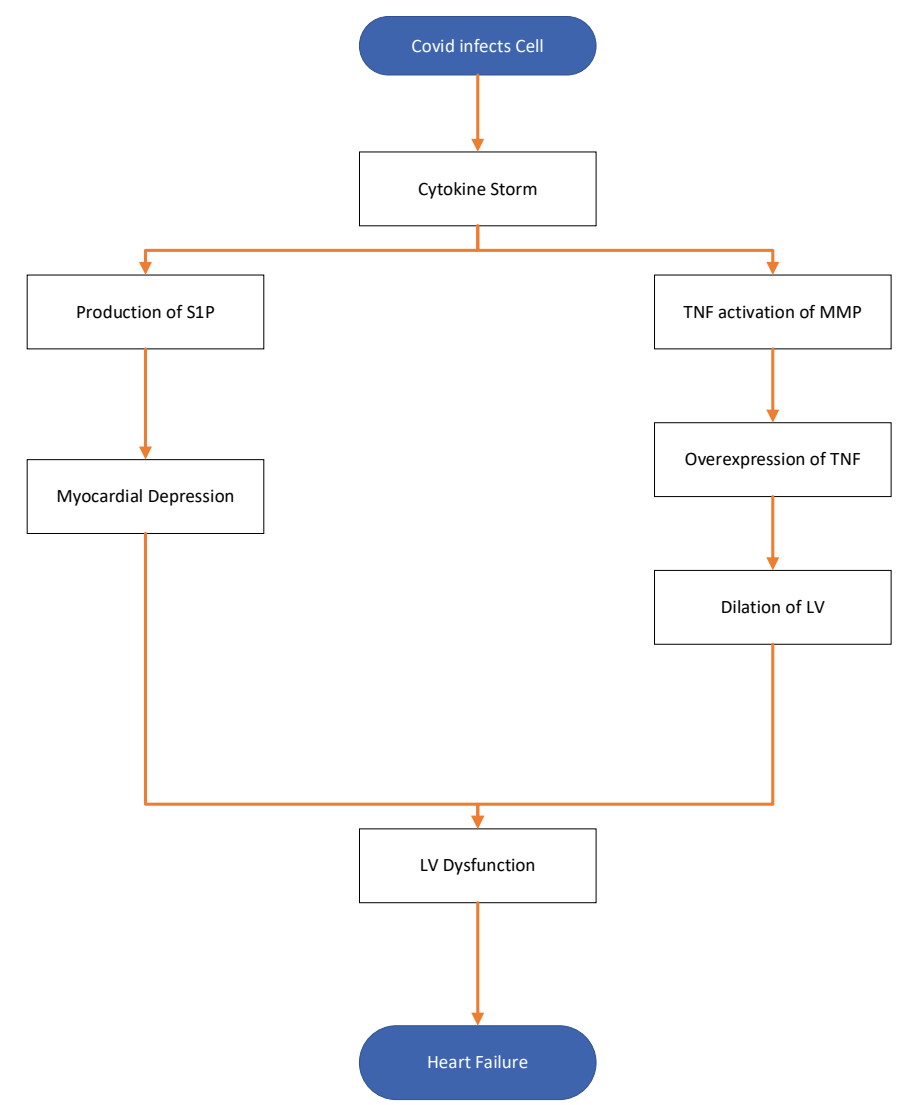

Fig. 4 Cytokines storms, associated with overaction of the immune system instigate excess production of S1P and TNF activation of MMP. Excess production of S1P induces myocardial depression, prompting LV dysfunction. TNF activation causes an overexpression of TNF, stimulating dilation of LV, resulting in LV dysfunction. LV dysfunction incites Heart failure

Cytokine Storms are commonly associated with Sars-Cov-2. When a Cell gets infected, it activates a large number of white blood cells, which includes natural killer cells, T-cells, dendritic cells, B-cells, macrophages, and monocytes. This activation releases inflammatory Cytokines through receptor-ligand interactions. The discharge of inflammatory cytokines activates a higher white blood cell count through a Positive Feedback Loop. After the pathogenic trigger, the repair process starts which can cause one of two results:

1. Gradual restoration of organ function

2. Fibrosis occurs in healing leading to persistent organ malfunction

This process can lead to a direct effect of Cytokines on the heart, or an indirect effect, with Cytokines causing myocardial Depression. In terms of Myocardial Depression, it is believed to be caused by the production of Sphingosine 1-phosphate(S1P) in Cytokines. S1P moderates cell fate and inflammation in both the physiological and pathological conditions.

Cytokines direct effect on the heart was found to be the result of the overexpression of Tumor necrosis factor (TNF). TNF regulates cardiac function through two pathways: (1) "Immediate pathway that is manifest within minutes and is mediated by activation of the neutral sphingomyelinase pathway" (Douglass L. Mann, 2015, 1254-1268), 
followed by (2) "A delayed pathway that requires hours to days to develop and is mediated by NO-mediated blunting of $\beta$-adrenergic signaling" (Douglass L. Mann, 2015, 1254-1268).

Research shows that activation of matrix metalloproteinases (MMPs) via Tumor Necrosis Factor (TNF) causes an overexpression of the TNF cytokine. Studies done in transgenic mice found that targeted overexpression of TNF cytokines in the heart resulted in a depressed Left Ventricle ejection. TNF promotes the remodeling of LV through alterations in the extracellular matrix. Researchers found that TNF causes a time-dependent modification in Left Ventricle size resulting in progressive deterioration of the extracellular matrix. Other studies done in mice also found that it was possible to develop LV dilation. Kubota et al observed the overexpression of TNF over a 24-week period and found that it led to progressive dilation of the Left Ventricle.

\section{Septic Shock}

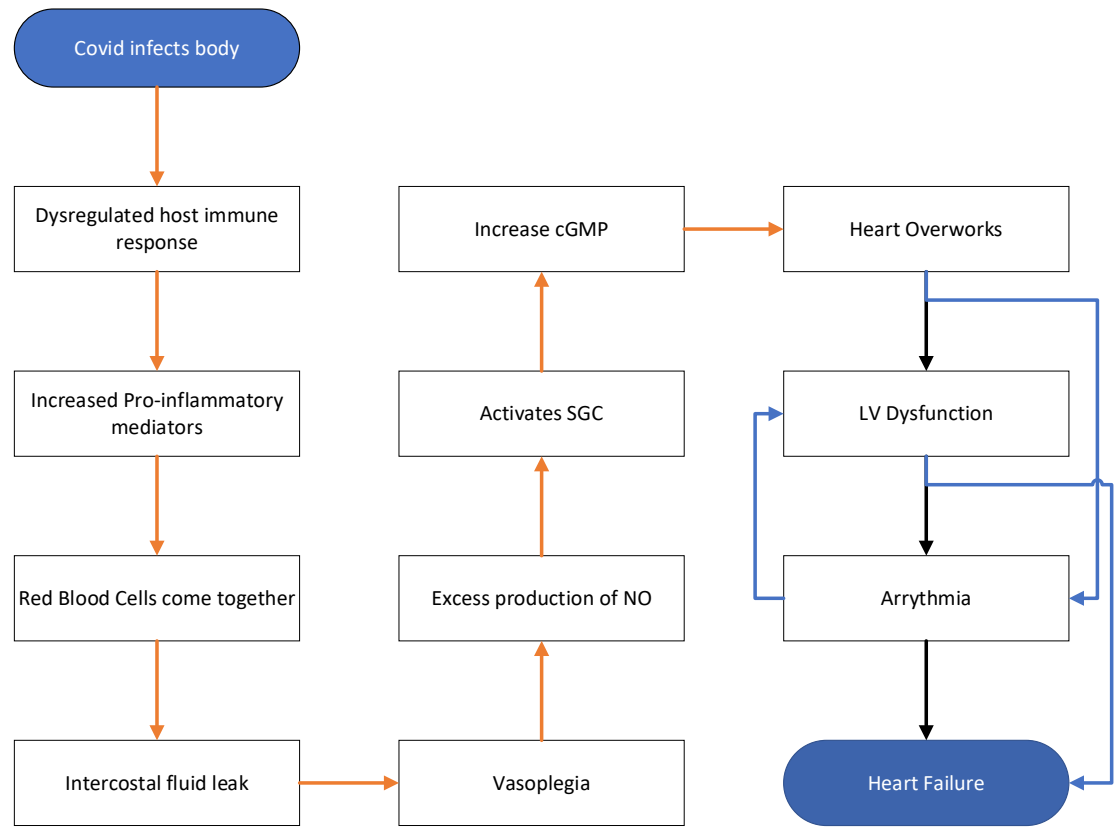

Fig. 5 Flowchart portrays the pathway by which Sars-Cov-2 induces Heart failure through sepsis and septic shock. Sars-Cov-2 causes an overaction of the immune system resulting in fluid leaks, excess of production of Nitric Oxide, activation of soluble guanylate cyclase, increase in cyclic guanosine monophosphate, LV Dysfunction and Arrythmia. The heart being overworked can result in arrythmia causing LV dysfunction and Heart failure, as shown by the blue arrows; or it can result in LV dysfunction, causing Arrythmia and Heart failure.

Sepsis is the body's overreaction to an infection triggering inflammation throughout the body. There are two types of sepsis: bacterial sepsis, the most studied and more commonly seen version, and viral sepsis, the understudied version that is not as commonly seen. Covid can cause viral sepsis, an indirect response to Sars-Cov-2 due to the virus's presence and not the virus infecting the tissue. Covid-induced viral sepsis is caused by the host's dysregulation response to the virus (the host attacks the body). The dysregulated response results in an increased pro-inflammatory mediator response (as commonly seen in other conditions caused by Sars-Cov-2). The increased pro-inflammatory mediator response brings together the red blood cells, compromising the microvascular blood flow. The compromised blood flow leads to fluid leaks in intercostal spaces and surrounding tissues, resulting in Septic Shock. Septic Shock is a condition in which sepsis is complicated by either hypotension that is refractory to fluid resuscitation (the compromised microvascular blood flow) or by hyperlactatemia. 
A common middle ground between Septic Shock and Heart failure is Left Ventricular dysfunction. Left Ventricular dysfunction is when the left ventricle heart chamber is damaged or defective. There are many reasons for LV dysfunction including blockage of blood passage, oxidative stress, inflammatory actions, Vasoplegia and myocardial injury. Vasoplegia is what is known to be a common cause of LV dysfunction from Septic Shock. In the body's blood vessels, vascular resistance is used to maintain organ perfusion. Vasoplegia causes a persistent and irreversible hypotension leading to a loss of vascular tone occurring through a "multifactorial mechanism" indicating a disruption between vasoconstrictors and vasodilators.

Nitric Oxide Synthase (iNOS) plays a major factor in this process. Sepsis-induced iNOS brings about a rise in the production of NO, resulting in an activation of the soluble guanylate cyclase (sGC). The activation of sGC generates an increase of cyclic guanosine monophosphate (cGMP). The increase in cGMP triggers a relaxation of myocardial and vascular smooth muscle, resulting in persistent and irreversible hypotension.

This hypotension leads to an increase of the heart rate resulting in the heart becoming hypertrophied including the left ventricle. The hypertrophied heart develops arrhythmia, inducing Heart failure.

\section{Renal Impairment:}

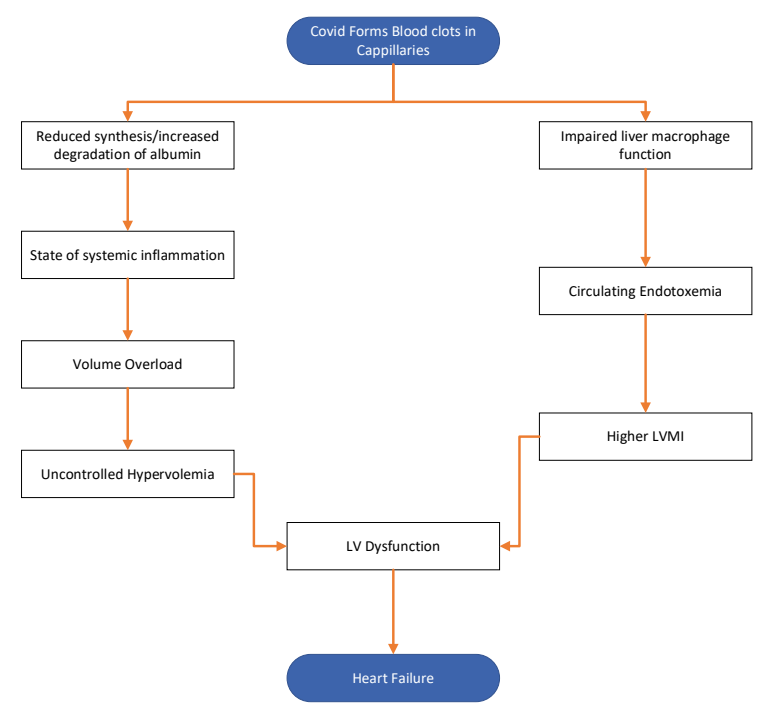

Fig. 6 Portrays the pathway by which Sars-Cov-2 stimulates Heart failure through renal impairment. Blood clots in the capillaries cause renal impairment, which lead to the other conditions shown in the figure. Patients with Sars-Cov2 gradually lose their ability to eject salt and water, along with a reduced synthesis and increased degradation of albumin, leading to a state of systemic inflammation, volume overload, uncontrolled hypervolemia, LV dysfunction, and Heart failure. The blood clot in the capillaries impair liver macrophage function, cause circulating endotoxemia, higher LVMI, LV dysfunction, and Heart failure.

Renal Impairment is caused by blood clots, formed by Sars-Cov-2, in the capillaries surrounding the kidneys. Patients with renal impairment steadily lose their capability to excrete salt and water. As renal impairments continually progress, there is a combination of reduced synthesis and increased degradation of albumin. This altered state of albumin has also been correlated to a state of systemic inflammation. The impairment of excretion of synthesis and water, combined with hypoalbuminemia (low level of albumin in blood) causes volume overload.

The increased sodium content in the body causes the body to attempt to balance the increased levels with water. However, the body produces too much water causing elevated plasma blood levels. Increase in interstitial pressure results in uncontrolled hypervolemia. This overload of pressure causes increased stress on the vein walls during systole resulting in LV dysfunction. 
Renal Impairment can also result in Endotoxemia. There is no definitive evidence as to how this occurs, but there is a theory that impaired liver macrophage function reduces endotoxin elimination. As a result, increased endotoxin presence in the blood circulates throughout the body. A collaboration of universities, including the University of Witwatersrand, did a study that found patients with elevated levels of circulating endotoxemia had a higher LVMI. In males, elevated levels of LVMI consist of greater than 115 grams per square meter, and in women, greater than 95 grams pers square meter.

\section{Sympathetic Activation}

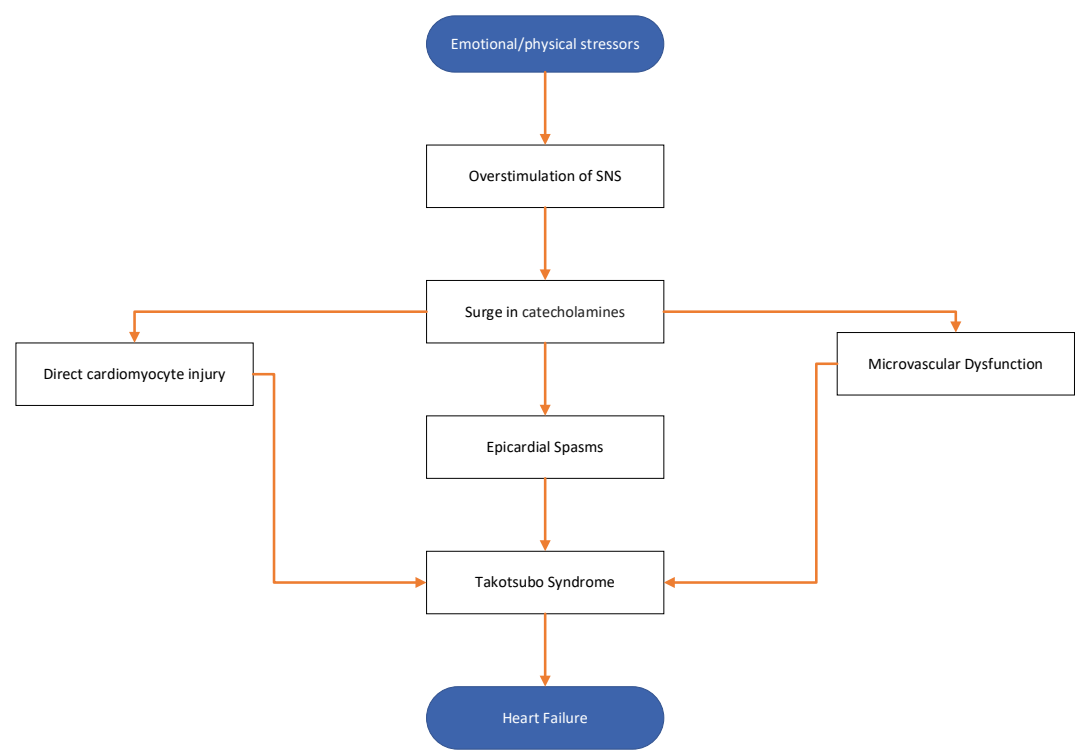

Fig. 7 Diagrams the process by which Sars-Cov-2 induces heart failure through the overstimulation of the Sympathetic Nervous System (SNS). Emotional/Physical stressors induce overstimulation of the SNS. Overstimulation stimulates a surge in catecholamines causing Direct cardiomyocyte injury, epicardial spasms, and microvascular dysfunction. Another complication of catecholamines surge is reversible stress cardiomyopathy (Takotsubo Syndrome). Enlargement of the ventricle caused by Takotsubo, a version of LV dysfunction, leads to heart failure.

Covid-19 causes sympathetic activation in multiple ways. Most common are emotional and physical distress, however, Covid can also cause changes in blood gases, angiotensin conversion enzyme imbalance, emotional distress, and increased production/release of Angiotensin II. Covid induced alterations have been associated with aggravated overactivation of the sympathetic nervous system.

The overactivation of the sympathetic nervous system has been discovered to cause Takotsubo. Takotsubo, which mainly affects postmenopausal women, is a specific type of LV dysfunction that is triggered by a strong emotional or physical stressor. It has been found that catecholamines are associated with this reversible stress-cardiomyopathy syndrome. The overstimulation of the sympathetic nervous system results in a surge of catecholamines, causing epicardial spasms, direct cardiomyocyte injury, and microvascular dysfunction. Especially in the apex of the heart, which was theorized to be more sensitive to the surge of Catecholamines caused by sympathetic stimulation.

\section{Acute Respiratory Disorder}




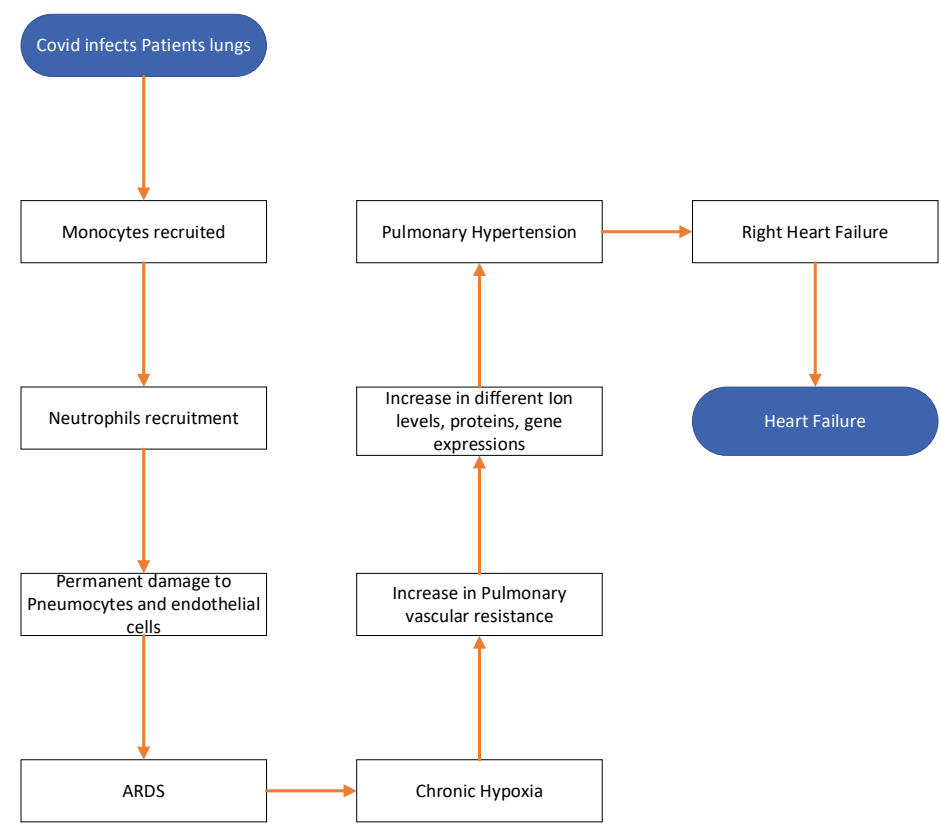

Fig. 8 Flow chart portrays the process by which Sars-Cov-2 induces Heart failure through ARDS. The recruited macrophages release chemokines and cytokines resulting in neutrophil's recruitment and increased capillary permeability. Induced ARDS triggers chronic hypoxia increased the pulmonary vascular resistance, prompting pulmonary hypertension, right heart failure, and Heart failure.

The membrane gas exchange region prevents air bubbles from forming in the blood and entering the alveolar. During ARDS, there is an "Acute and diffuse inflammatory damage into the alveolar-capillary barrier associated with a vascular permeability increase and reduced compliance, compromising gas exchange and causing hypoxemia" (Batah et all 2020, 106239).

Patients infected with Sars-Cov-2 secrete pro-inflammatory cytokines as a result of "recruited" monocytes in the alveolar space. The "recruited" macrophages also release chemokines and other cytokines, resulting in neutrophils recruitment and elevated capillary permeability. The excessive neutrophils degranulation causes permanent harm endothelial cells and pneumocytes, provoking a breakage in the alveolar-capillary barrier.

In a patient with ARDS, there is an elevation of pulmonary vascular resistance. Pulmonary hypertension is also known to be caused by chronic hypoxia which resulted from ARDS. The vascular wall is in a balance between proliferation and apoptosis. In patients with Pulmonary hypertension, the vessel wall thickens, destroying the vessel lumen causing increased resistance. The increased resistance causes vascular remodeling, leading to an increase in pulmonary pressure generating further vascular remodeling. This process is all caused by the reaction of the body's cells to hypoxia.

The cellular response to hypoxia has been noted to result in a dramatic increase of $\mathrm{Ca}^{2+}$ levels. The increase of this ion results in the activation of calmodulin, MAPks, and expression of the proto-oncogene c-fos. The increased levels of $\mathrm{Ca}^{2+}$ in SMC has also been shown to control growth and proliferation. In the end, the dramatic increase in different ion levels, as well as different proteins and gene expressions leads to vascular remodeling. The vascular remodeling also causes an increase in the muscle of the blood vessels going to the right ventricle, inducing increased blood pressure in the vessels. The blood pressure of these vessels on average are lower than both the diastolic and systolic pressure. Excessively high blood pressure in the vessels entering the right ventricle may trigger right heart failure.

Right heart failure is the right ventricles response to pulmonary hypertension. Due to the lack of substantial research in right heart failure, researchers cannot assess a definitive process from PH to RHF. However, researchers believe that patient has Pulmonary hypertension, the Right Ventricle increases the afterload. There are a multitude of 
responses that could result from increased afterload: hypertrophy of the RV, reduced coronary blood flow, dilation of the Tricuspid annulus, and displacement of the interventricular septum; all of which can result in Right Heart Failure.

\section{Conclusion}

Sars-Cov-2 is a virus that initially causes an imbalance in the ACE-1 and ACE-2 levels. The virus uses its receptor to attach to the ACE-2 causing increased blood pressure, resulting in a multitude of dangerous conditions. Patients who suffer from Covid-19 may experience metabolic health issues, Thrombosis, Ischemia, Direct cardiac injury, Myocarditis, inflammatory mediators, Myocardial depression, excessive inflammatory mediators such as cytokine storms, Septic shock, Renal impairment, Volume overload, Fluid overload, overactivation of the Renin-angiotensin system, Stress cardiomyopathy, Sympathetic activation, Acute respiratory distress syndrome, Pulmonary hypertension, right heart failure, Left ventricular dysfunction, and Heart failure. There are currently 4 vaccines available: Pfizer, Moderna, Johnson \& Johnson, and AstraZeneca. The Pfizer and Moderna vaccines both got emergency approval by the FDA and use a new concept of mRNA to give instructions to cells. There are currently no antiviral treatments available to the public, however, Pfizer is working on an antiviral which stops the replication of Sars-Cov-2 using protease inhibitors that bind to the viral enzymes.

\section{Acknowledgments}

I would like to recognize my mentor, Roel Mercado, for assisting me throughout my research. I would like to thank Dr. Maria Gomez for fact checking my paper, Dr. Suzanne Moeller for editing my paper, and Dr. Steven Barnhart for reviewing my paper for plagiarism.

\section{References}

1) Colantuoni, A., Martini, R., Caprari, P., Ballestri, M., Capecchi, P. L., Gnasso, A., .. Caimi, G. (2020). COVID-19 Sepsis and Microcirculation Dysfunction. Frontiers in Physiology, 11. doi:10.3389/fphys.2020.00747

2) Clinic, C. (n.d.). Heart Failure: Types, Symptoms, Causes \& Treatments. Retrieved June 3, 2021, from https://my.clevelandclinic.org/health/diseases/17069-heart-failure-understanding-heart-failure\#management-and-treatment

3) L. (n.d.). The Pericardium. Retrieved from https://www.liverpool.ac.uk/ trh/local html/heartdisease/pericardium.htm

4) M. (n.d.). Chambers and valves of the heart. Retrieved July 24, 2021, from https://www.mayoclinic.org/chambers-and-valves-of-the-heart/img-20007497

5) Ayres, J.S. A metabolic handbook for the COVID-19 pandemic. Nat Metab 2, 572-585 (2020). https://doi.org/10.1038/s42255-020-0237-2

6) Lupi, L., Adamo, M., Inciardi, R. M., \& Metra, M. (2020). ACE2 down-regulation may contribute to the increased thrombotic risk in COVID-19. European Heart Journal, 41(33), 3200-3200. doi:10.1093/eurheartj/ehaa583

7) Clinic, C. (n.d.). Deep Vein Thrombosis (DVT); Symptoms, Causes, Treatment \& Prevention. Retrieved June 3, 2021, from https://my.clevelandclinic.org/health/diseases/16911-deep-vein-thrombosis-dvt

8) Rodriguez, B., Trayanova, N., \& Noble, D. (2006). Modeling Cardiac Ischemia. Annals of the New York Academy of Sciences, 1080(1), 395-414. doi:10.1196/annals.1380.029

9) Blaisdell FW, Steele M, Allen RE. Management of acute lower extremity arterial ischemia due to embolism and thrombosis. Surgery. 1978 Dec;84(6):822-34. PMID: 715701. 
10) Vitiello, A., \& Ferrara, F. (2020). Pharmacological agents to therapeutic treatment of cardiac injury caused by Covid-19. Life Sciences, 262, 118510. doi:10.1016/j.1fs.2020.118510

11) Fara, A., Mitrev, Z., Rosalia, R. A., \& Assas, B. M. (2020). Cytokine storm and COVID-19: A chronicle of pro-inflammatory cytokines. Open Biology, 10(9), 200160. doi:10.1098/rsob.200160

12) Al, A. N. (2020). Cardiac and arrhythmic complications in Covid-19 patients. Authorea. doi:10.22541/au.158594433.36475644

13) Matsumori, A. (2000). Cytokines and Heart Failure: Pathophysiological Roles and Therapeutic Implications. Heart Failure, 35-45. doi:10.1007/978-4-431-68331-5_3

14) Meacci, E., Garcia-Gil, M., \& Pierucci, F. (2020). SARS-CoV-2 Infection: A Role for S1P/S1P Receptor Signaling in the Nervous System? International Journal of Molecular Sciences, 21(18), 6773. doi:10.3390/ijms21186773

15) Mann, D. L. (2001). The Role of Inflammatory Mediators in the Failing Heart. Developments in Cardiovascular Medicine The Role of Inflammatory Mediators in the Failing Heart, 1-1. doi:10.1007/978-1-46151449-7_1

16) Guo BB, Bellingham $S A$, Hill AF. The neutral sphingomyelinase pathway regulates packaging of the prion protein into exosomes. J Biol Chem. 2015 Feb 6;290(6):3455-67. doi: 10.1074/jbc.M114.605253. Epub 2014 Dec 10. PMID: 25505180; PMCID: PMC4319014.

17) Alliance, S. (2020, March 26). The Connection Between COVID-19, Sepsis, and Sepsis Survivors. Retrieved June 3, 2021, from https://www.sepsis.org/news/the-connection-between-covid-19-sepsis-and-sepsis-survivors/

18) Shappell, C. N., Klompas, M., \& Rhee, C. (2020). Does Severe Acute Respiratory Syndrome Coronavirus 2 Cause Sepsis?. Critical care medicine, 48(12), 1707-1709. https://doi.org/10.1097/CCM.0000000000004601

19) Angus, D. C., \& Poll, T. V. (2013). Severe Sepsis and Septic Shock. The New England Journal of Medicine. doi:10.1056/NEJMra1208623

20) NHS. "Septic Shock Symptoms and Treatment." Illnesses \& Conditions | NHS Inform. February 10, 2020. Accessed June 03, 2021. https://www.nhsinform.scot/illnesses-and-conditions/blood-and-lymph/septicshock.

21) Huang, S.J., Nalos, M. \& McLean, A.S. Is early ventricular dysfunction or dilatation associated with lower mortality rate in adult severe sepsis and septic shock? A meta-analysis. Crit Care 17, R96 (2013). https://doi.org/10.1186/cc12741

22) Gamcrlidze MM, Intskirveli NA, Vardosanidze KD, et al. Vasoplegia in septic shock (review). Georgian Medical News. 2015 Feb(239):56-62.

23) Sharawy, Nivin. "Vasoplegia in Septic Shock: Do We Really Fight the Right Enemy?" ScienceDirect, February 2014. Accessed June 3, 2021. doi:10.1016/j.jcrc.2013.08.021.

24) Stasch, J. P., Pacher, P., \& Evgenov, O. V. (2011). Soluble guanylate cyclase as an emerging therapeutic target in cardiopulmonary disease. Circulation, 123(20), 2263-2273. https://doi.org/10.1161/CIRCULATIONAHA.110.981738

25) 1. Burgdorff A-M, Bucher M, Schumann J. Vasoplegia in patients with sepsis and septic shock: pathways and mechanisms. Journal of International Medical Research. April 2018:1303-1310. doi:10.1177/0300060517743836

26) Sperati, C. J. (n.d.). Coronavirus: Kidney Damage Caused by COVID-19. Retrieved June 3, 2021, from https://www.hopkinsmedicine.org/health/conditions-and-diseases/coronavirus/coronavirus-kidney-damagecaused-by-covid19\#: :text=COVID-19 causes blood clots,kidney and impair its function

27) Ebah, L. M., Wiig, H., Dawidowska, I., Otoole, C., Summers, A., Nikam, M., . . Mitra, S. (2013). Subcutaneous interstitial pressure and volume characteristics in renal impairment associated with edema. Kidney International, 84(5), 980-988. doi:10.1038/ki.2013.208 
28) Nisbeth U, Hällgren R, Eriksson O, Danielson BG. Endotoxemia in chronic renal failure. Nephron. 1987;45(2):93-7. doi: 10.1159/000184086. PMID: 3550501.

29) F. (n.d.). Hypervolemia and Signs of Fluid Overload. Retrieved June 3, 2021, from https://www.freseniuskidneycare.com/thrive-central/hypervolemia

30) Hassan, M. O., Duarte, R., Dix-Peek, T., Vachiat, A., Naidoo, S., Dickens, C., . . Naicker, S. (2016). Correlation between volume overload, chronic inflammation, and left ventricular dysfunction in chronic kidney disease patients. Clinical Nephrology, 86(S1), 131-135. doi:10.5414/cnp86s127

31) Sperati, C. (n.d.). Coronavirus: Kidney Damage Caused by COVID-19. Retrieved June 3, 2021, from https://www.hopkinsmedicine.org/health/conditions-and-diseases/coronavirus/coronavirus-kidney-damagecaused-by-covid19

32) Porzionato, A., Emmi, A., Barbon, S., Boscolo-Berto, R., Stecco, C., Stocco, E., . . Caro, R. D. (2020). Sympathetic activation: A potential link between comorbidities and COVID-19. The FEBS Journal, 287(17), 3681-3688. doi:10.1111/febs.15481

33) Sverrisdóttir, Y. B., Schultz, T., Omerovic, E., \& Elam, M. (2012). Sympathetic nerve activity in stressinduced cardiomyopathy. Clinical autonomic research : official journal of the Clinical Autonomic Research Society, 22(6), 259-264. https://doi.org/10.1007/s10286-012-0162-x

34) Wittstein, I. S. (2016). The Sympathetic Nervous System in the Pathogenesis of Takotsubo Syndrome. ResearchGate. http://dx.doi.org/10.1016/j.hfc.2016.06.012

35) Batah, S. S., \& Fabro, A. T. (2021). Pulmonary pathology of ARDS in COVID-19: A pathological review for clinicians. Respiratory Medicine, 176, 106239. doi:10.1016/j.rmed.2020.106239

36) C. (2019, December 03). Pulmonary Hypertension. Retrieved June 3, 2021, from https://www.cdc.gov/heartdisease/pulmonary hypertension.htm

37) Pak, O., Aldashev, A., Welsh, D., \& Peacock, A. (2007). The effects of hypoxia on the cells of the pulmonary vasculature. European Respiratory Journal, 30(2), 364-372. doi:10.1183/09031936.00128706

38) Bogaard, H., Abe, K., Noordegraaf, A., \& Voelkel, N. (2009). The Right Ventricle Under Pressure: Cellular and Molecular Mechanisms of Right-Heart Failure in Pulmonary Hypertension. ScienceDirect. doi:https://doi.org/10.1378/chest.08-0492

39) Guarracino F, Cariello C, Danella A, Doroni L, Lapolla F, Vullo C, Pasquini C, Stefani M. Right ventricular failure: physiology and assessment. Minerva Anestesiol. 2005 Jun;71(6):307-12. PMID: 15886593. 